\title{
Wie ein kalter Händedruck Medizingeschichte schrieb
}

\section{Ronald Gerste}

Dr. med., Dr. phil., Facharzt für Augenheilkunde, Medizinhistoriker und Journalist

An die erste Patientin mit dieser ganz speziellen Symptomatik erinnert sich Professor Josef Flammer noch genau. Es war Anfang der 1980er Jahre, und Flammer war Assistent an der Universitätsaugenklinik in Bern: «Sie war Mitte Vierzig und hatte ein Normaldruckglaukom. Besonders auffallend: Sie hatte sehr kalte Extremitäten, und wir begannen uns zu fragen, ob es einen Zusammenhang mit dem Augenbefund gab. Wir fan-

\section{«Sie hatte sehr kalte Extremitäten, und wir begannen uns zu fragen, ob es einen Zusam- menhang mit dem Augenbefund gab.»}

den bei einer Spezialuntersuchung ihrer Hände, einer Kapillarmikroskopie bei den angiologischen Kollegen, schnell heraus, dass sie unter sogenannten Vasospasmen litt: Die kleinen Blutgefässe in der Peripherie - das können die Finger sein oder auch das Ohr, das Auge krampfen sich als Reaktion auf einen Stimulus wie Kälte oder Stress regelrecht zusammen.»

Flammer konnte nicht ahnen, dass der vermeintliche Nebenbefund, der ihm bereits aufgefallen war, als er die Patientin mit einem Händedruck willkommen geheissen hatte, ihn auf eine Spur führte, die in sein wissenschaftliches und klinisches Lebenswerk mündete - und dazu, dass er zur seltenen Spezies von Medizinern wurde, die bereits zu Lebzeiten mit einem ihren Namen tragenden Syndrom Einzug in die medizinische Terminologie halten sollten. Der lange Weg zu einem weltweit anerkannten Spezialisten, dessen Forschungen Bedeutung weit über sein eigenes Fachgebiet hinaus haben, hat einen Ursprung, wie er rustikaler kaum sein kann. Es ist gleichzeitig eine Vita, welche die positivsten Klischees über die Schweiz zu bestätigen scheint: dass auch bei Herkunft aus den sogenannten einfachen Verhältnissen der Aufbruch in die Welt jenseits der von schneebedeckten Gipfeln gesäumten Horizonte, der Aufstieg auf eine globale Bühne (in diesem Fall jene der Wissenschaft) stets möglich ist; Fleiss, Redlichkeit und Charakterstärke vorausgesetzt.

Josef Flammer wurde 1948 auf einem Bauernhof in der Nähe von Bronschhofen in der Ostschweiz geboren. «In

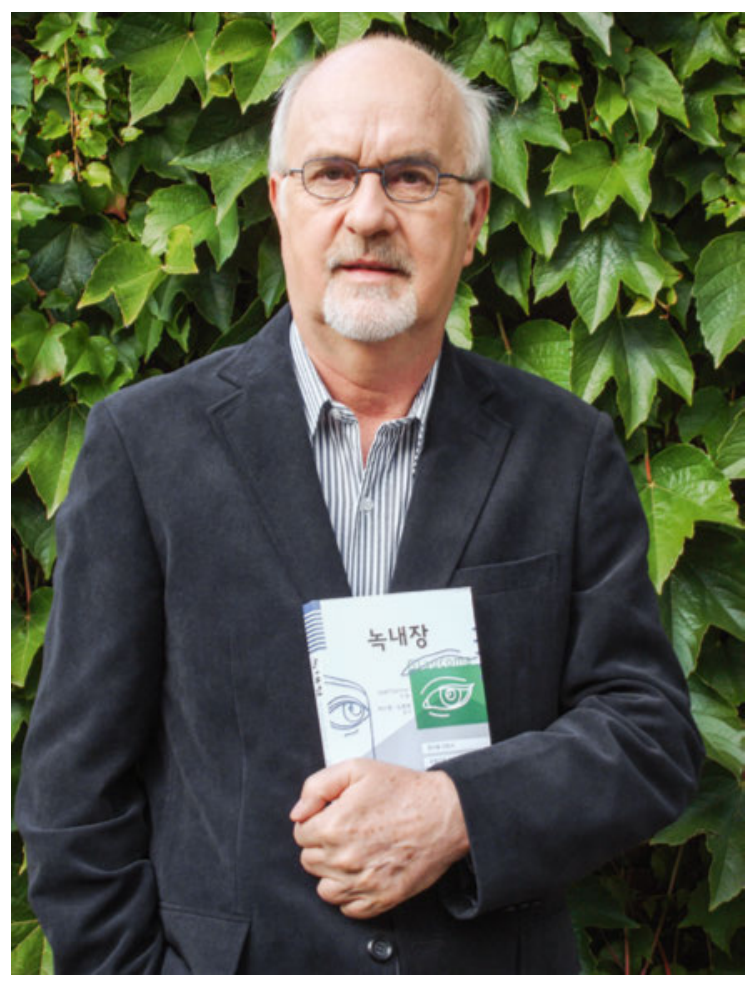

Er erkannte bei seiner Patientin den Zusammenhang zwischen ihrem kalten Händedruck und dem Normaldruckglaukom: Prof. em. Dr. Josef Flammer.

den dortigen lokalen Verhältnissen», so erinnert sich Flammer, «waren wir eine kleine Familie; ein Nachbar hatte 13, ein anderer 14 Kinder. Meine Mutter hatte sich fast gescheut, weil sie nur 5 Kinder hatte; wir Geschwis-

Die Kinder fuhren manchmal kilometerweit mit dem Velo, um ein Kuvert zu überbringen und damit die Briefmarke einzusparen.

ter waren 2 Mädchen und 3 Buben.» Er wuchs in harmonischen, wenn auch armen Verhältnissen auf; Schuhe wurden gebraucht getragen, die Kinder fuhren manchmal kilometerweit mit dem Velo, um ein Kuvert zu überbringen und damit die Briefmarke einzusparen. Josef Flammer besuchte schliesslich ein Internat in Gossau und nahm 1968 das Medizinstudium auf, zunächst in Fribourg und dann in Bern. Seine Assisten- 
tenzeit absolvierte er in den Fächern Innere Medizin, Neurologie und Augenheilkunde, die später allesamt eine Bedeutung für die Erforschung der letztlich seinen Namen tragenden Symptomatiken bekamen. Letztlich entschied er sich für die Augenheilkunde, in die er sich im kanadischen Vancouver und in Bern vertiefte, wo er Oberarzt wurde - und die recht junge Akademikerin, quasi Patientin Zero, mit den kalten Händen betreute. Nachdem er 1987 den Ruf als Direktor der Universitätsaugenklinik Basel angenommen hatte, wurde die Klinik in Patientenbetreuung, Weiterbildung Flammers zunächst im Frühling, dann im November

Er entschied sich für die Augenheilkunde, in die er sich im kanadischen Vancouver und in Bern vertiefte.

stattfindendes "Basler Glaucoma-Meeting» avancierte schnell zu einer der angesehensten Tagungen des Faches in Mitteleuropa - und Forschung zur "Glaukomhochburg». In jenen Jahren setzte sich unter Augenärzten die ein wenig verunsichernde Erkenntnis durch (ganz wesentlich aufgrund Basler Studien und Publikationen), dass der "Grüne Star» keineswegs immer allein durch den Augeninnendruck auf die überkommene und scheinbar so eindeutige Art zu definieren ist: Unter $22 \mathrm{~mm} \mathrm{Hg}$ gesund, über $22 \mathrm{~mm}$ Hg krank. Man erkannte die Bedeutung und weit unterschätzte Prä-
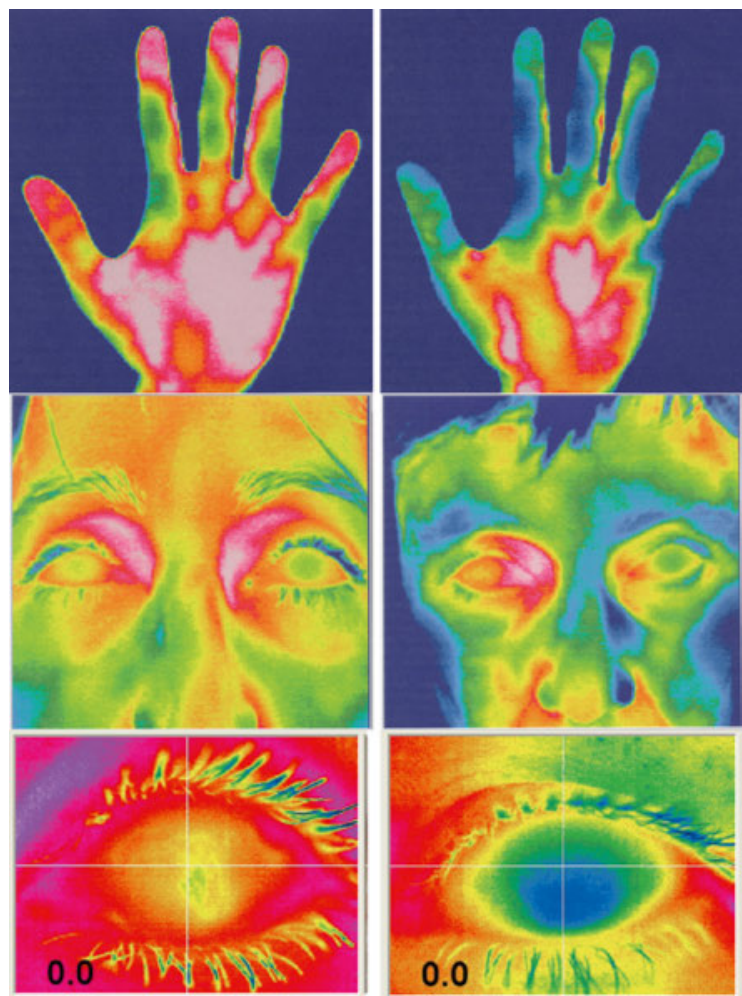

Bild von einer Thermographie.

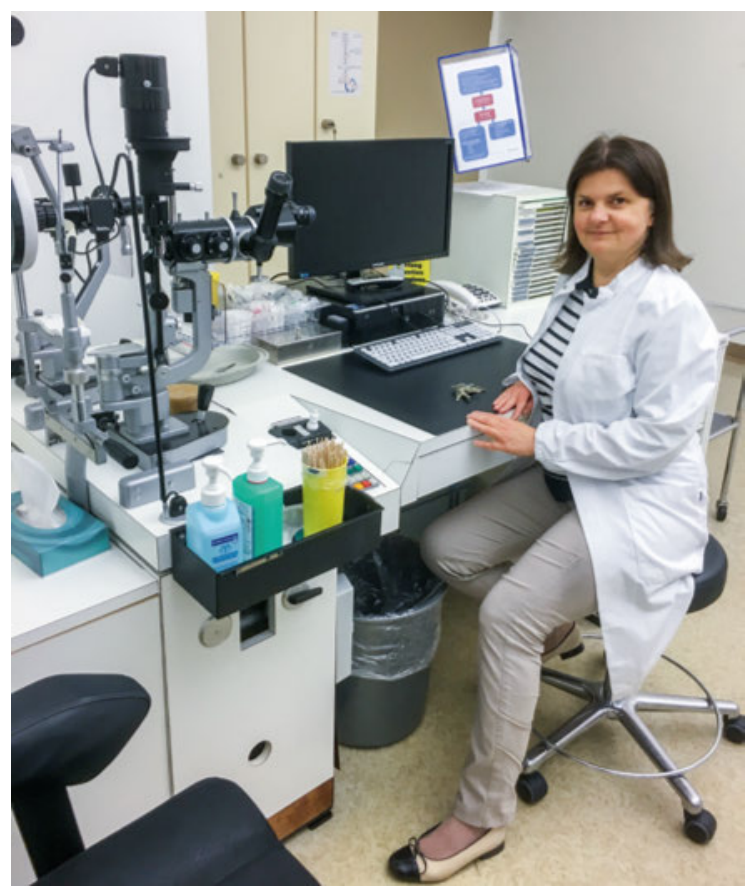

Der Augenärztin Katarzyna Konieczka und ihren Kollegen ist es zu verdanken, dass der Begriff des Flammer-Syndroms nun offiziell in der Fachliteratur zu finden ist.

valenz des Normaldruckglaukoms - diese Patienten haben einen scheinbar "gesunden" Augeninnendruck von vielleicht 16, vielleicht $18 \mathrm{~mm} \mathrm{Hg}$, aber die klinischen Merkmale dieser Optikusneuropathie wie Papillenexkavation und progrediente Gesichtsfeldausfälle. Und viele Normaldruckglaukompatienten haben noch mehr: Kalte Hände. Einschlafstörungen. Tinnitus. Manchmal auch Migräne. Und vor allem: arteriellen Hypotonus.

Flammer, der Gesichtsfeldindices in die augenärztliche Diagnostik einführte und das Konzept des Reperfusionsschadens beim Glaukom entwickelte, begann Ende der 1980er Jahre Untersuchungen mit Instrumenten einzuführen, die wahrlich nicht zur augenärztlichen Standardausrüstung gehören: mit der Nagelfalzmikroskopie, später mit der Doppler-Sonographie und der Thermographie. Der reduzierte Blutfluss in dieser äussersten Körperperipherie passte in ein sich allmählich entwickelndes Gesamtbild, zu dem ein systemischer Hypotonus gehört. Die nächtlichen «Dips» eines möglicherweise schon tagsüber recht niedrigen Blutdrucks wurden von Flammer und Mitarbeitern als ein wesentliches Risiko für die Ganglienzellen in Retina und Sehnerv des Normaldruckglaukompatienten identifiziert. Basel wurde zu einer Art Wallfahrtsstätte für Menschen - in Flammers späten Jahren dort aus buchstäblich fünf Kontinenten -, denen die Ärzte nicht helfen konnten, vor allem da ein interdisziplinärer Ansatz fehlte. Der Augenarzt behandelte bei ihnen das Glau- 
kom und der Internist den tiefen Blutdruck. In Basel wurde versucht, all diese so auffallend oft gemeinsam auftretenden Symptome in einem Zusammenhang zu sehen und, wo es nötig ist, zu behandeln.
Korrespondenz: Dr. med. Ronald Gerste rdgerste@aol.com
Auf internationalen Kongressen wurde deutlich, dass die bestehende Terminologie für den Symptomenkomplex nicht richtig zufriedenstellte. Weder das zunächst gebräuchliche "vasospastische Syndrom» noch "primäre vaskuläre Dysregulationen (PVD)» trafen es auf den Punkt. Eine internationale Expertengruppe unter der Federführung einer in der Schweiz tätigen polnischen Augenärztin, Katarzyna Konieczka, hat dann den Begriff des Flammer-Syndroms offiziell in die Fachliteratur eingeführt. Dieser Terminus hat sich ab etwa 2013 fast schlagartig durchgesetzt und ausgebreitet. Flammer unterstreicht, dass ein Syndrom nicht gleichbedeutend mit Morbidität sein muss: «Denn ein Flammer-Syndrom zu haben, bedeutet noch lange nicht krank zu sein. Menschen mit FlammerSyndrom haben gewisse Krankheiten sogar seltener, andere dafür häufiger. Seltener sind beispielsweise die Arteriosklerose und ihre Folgen.»

Seit seiner Emeritierung vor vier Jahren ist Flammer dem vor kurzem der "EPMA Award of Excellence in Research" verliehen wurde - ungebrochen wissenschaftlich und publizistisch aktiv, Einladungen für Vorträge und keynote lectures führen ihn nach China, Russland und Amerika. Betritt er auf seinen Reisen eine Buchhandlung mit Schwerpunkt auf medizinischer Literatur, trifft er häufig auf etwas Bekanntes: Sein Standardwerk über das Glaukom (mit einem grossen Segment über vaskuläre Aspekte) für Mediziner wie gebildete Laien und Betroffene ist inzwischen in 24 Sprachen übersetzt worden, darunter Amharisch (Äthiopien), Koreanisch, Georgisch und in einem pakistanischen Idiom. In einem Nachbarland des Letzteren, in Indien, ist gerade ein Screeningprogramm eingeführt worden: Bei Schülern zwischen 8 und 18 Jahren wird nach Anzeichen von Flammer-Syndrom und oxidativem Stress gesucht.

\section{Bildnachweise}

Foto von Prof. Flammer: $@$ Josef Flammer

Foto von Katarzyna Konieczka: @ Katarzyna Konieczka Bild «Thermography»: aus Flammer J. Glaucoma. 3rd edition. Bern: Hogrefe \& Huber; 2006

Bild «Häufige Symptome des Flammer-Syndroms»: aus Konieczka K, Flammer J. Phänomenologie und klinische Bedeutung des FlammerSyndroms. Klin Monbl Augenheilkd. 2016;233(12):1331-6. 